\title{
Mehr Patientensicherheit durch Design
}

\author{
Lynn Häsler, Irene Kobler
}

Patientensicherheit Schweiz

\author{
Wie kann man Patientensicherheit in ein Spital "hineindesignen»? Wird Ver- \\ hältnisprävention in der Patientensicherheit vernachlässigt? Diese Fragen warf das \\ Symposium «Mehr Patientensicherheit durch Design» der Stiftung Patienten- \\ sicherheit am 7. April 2017 im Careum Auditorium in Zürich auf.
}

Die Stiftung Patientensicherheit Schweiz lanciert eine Debatte um ein noch wenig beachtetes Thema. «Der Professionelle soll seine Aufgabe wahrnehmen können.» Prof. Dr. Dieter Conen, Präsident der Stiftung

\section{Wären Fehler eine Krankheit, befänden sie sich unter den Top 10.}

Patientensicherheit, appelliert an die Verantwortung von Organisationen gegenüber ihren Mitarbeitern. «Es gibt ein systemisches, strukturelles Problem in der Patientensicherheit», erinnert Prof. Dr. David Schwap-

\section{Plus de sécurité des patients grâce au design}

Comment peut-on intégrer la sécurité des patients dans le design d'un hôpital? Cette question était au centre du symposium «Plus de sécurité des patients grâce au design" du 7 avril 2017 à I'auditorium Careum à Zurich. De nombreux experts issus de divers secteurs professionnels affirment que le design influe sur la guérison des patients, et oriente également le comportement des collaborateurs par le biais de l'environnement de travail. Le design a donc aussi une influence sur la sécurité des patients. Un environnement aménagé spécifiquement peut aider à rendre les actes plus sûrs et les erreurs moins probables. Ce n'est finalement pas étonnant que des erreurs soient commises si les collaborateurs doivent essayer de se concentrer dans des conditions d'éclairage déficientes ou un environnement sonore. Même si le problème est connu depuis longtemps, on ne s'occupe pas assez de prévention. Si la formation est efficace, elle ne sert toutefois pas à grand-chose lorsque les conditions de travail ne permettent pas de la mettre en œuvre. Le principe du design comme aide à l'amélioration de la sécurité des patients n'est que peu répandu dans la pratique, c'est pourquoi la fondation Sécurité des patients Suisse a lancé le projet «Plus de sécurité des patients grâce au design: solutions systémiques pour l'hôpital». Lors du premier symposium suisse sur ce sujet, des expertes et experts internationaux ont proposé de nouvelles approches. pach, wissenschaftlicher Leiter der Stiftung Patientensicherheit. Wären Fehler eine Krankheit, befänden sie sich unter den Top 10. Obwohl das Problem seit 20 Jahren bekannt ist, wird Verhältnisprävention vernachlässigt und vor allem auf Training gesetzt. Training ist wirksam, nützt aber nichts, wenn die Arbeitsverhältnisse daran hindern, das Trainierte umzusetzen. «Es geht auch um Wertschätzung des Spitals gegenüber den Mitarbeitenden", findet Schwappach.

\section{Spezialisten für förderliches Design gibt es bereits}

Prof. Dr. Craig Zimring, Psychologe und Professor für evidence-based health care design an der School of Architecture des Georgia Institute of Technology, formuliert pointiert: «Schlechtes Spitaldesign macht uns kränker.» Er präsentierte zahlreiche eindrucksvolle Beispiele, in denen die Architektur und das Design von Spitälern die Versorgungsqualität beeinflussen. So unterscheidet sich die Mortalitätsrate in Krankenzimmern, in denen die Patienten für das Personal vom Gang aus schlecht sichtbar sind, systematisch von den Raten in anderen Zimmern. Dass die Überlebenswahrscheinlichkeit davon beeinflusst ist, in welchem Zimmer man untergebracht ist, sei inakzeptabel. Im Spitaldesign sei es ausschlaggebend, von den Automatismen, die die Handlungen im Alltag lenken, Gebrauch zu machen. Denn spätestens seit Daniel Kahnemans Forschung zu den verschiedenen Arten des Denkens ist anerkannt, dass Menschen nicht immer rational agieren. Man muss dieses vermeintliche Manko daher geschickt ausnützen und Spitäler so designen, dass man «automatisch» das Richtige tut - sei es nur, indem man die Sichtbarkeit der Desinfektionsspender erhöht. «Use design to prevent smart people from doing foolish things.» Lenny Naar, Design-Stratege am Helix 
Centre am Imperial College in London, setzt auf interdisziplinäre Teams. Wenn Designer, Spitalmitarbeiter, Patienten, Akademiker und Ingenieure zusammenarbeiten, können Probleme von allen Seiten beleuchtet werden. Lösungen haben allerdings nur dann eine Chance, wenn sie eine attraktive Handlungsoption darstellen, bei der sich die Ausführenden nicht unbehaglich oder gar albern vorkommen. Die bewährten Methoden, um den Bedürfnissen und Motiven auf den Grund zu gehen, seien Empathie und Prototyping. Neues mit Vertrautem und Bewährtem zu kombinieren sei zudem ein gutes Rezept, um die Akzeptanz von neuen Lösungen zu erhöhen. Fachinformationen können zum Beispiel im Facebook-Stil präsentiert werden. Hannah Behrendt vom «Behavioural Insights Team» in London, das die Regierung Grossbritanniens dabei unterstützt, durch "Nudging» (engl. Schubsen, Anstossen) erwünschtes Verhalten auf unaufdringliche Art zu fördern, ergänzt, dass Lösungen nicht nur attraktiv, sondern auch einfach sein müssen und das Verhalten sozial erwünscht sein muss. Auch Timing spielt eine Rolle. «Unbewusstes Verhalten kann nicht trainiert werden.»

\section{Den Stein ins Rollen bringen}

Irene Kobler leitete das Projekt und stellte die Meilensteine und die Broschüre zum Thema vor. Ziel von Patientensicherheit Schweiz ist es, die Verantwortlichen für systemische Lösungen im Bereich Design und $\mathrm{Pa}$ tientensicherheit zu sensibilisieren und das Thema auf

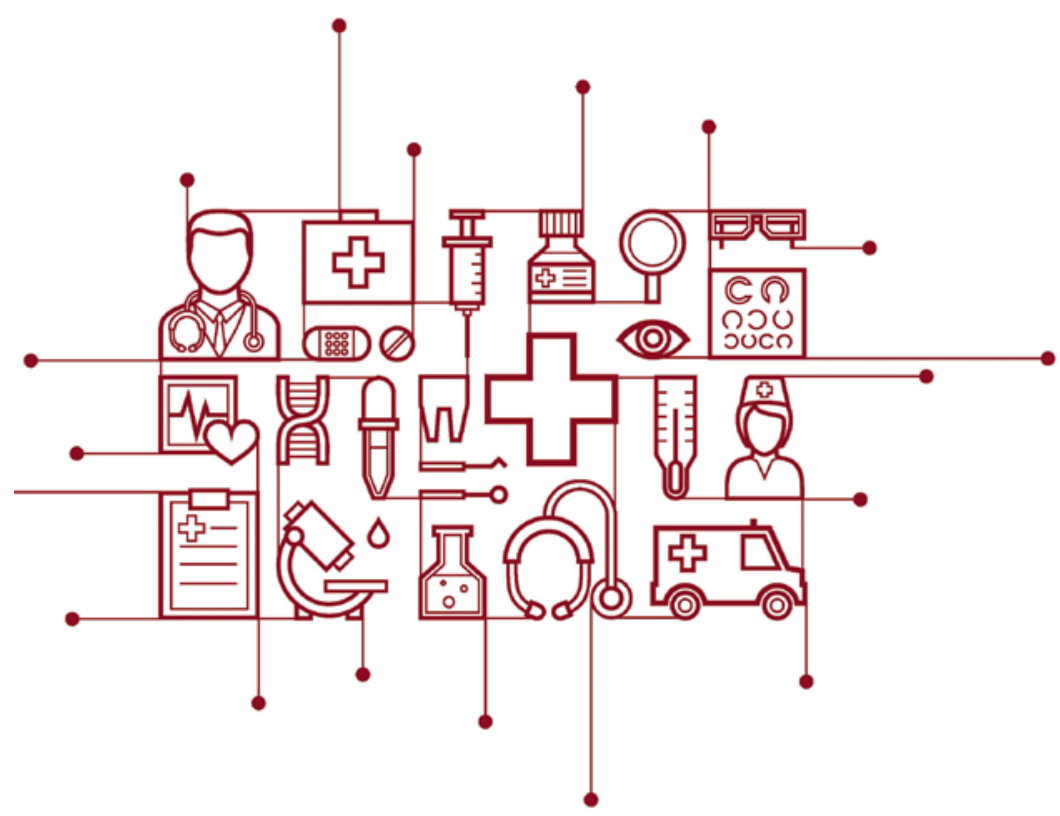

Welche Rolle spielt das Design für die Patientensicherheit? Am Symposium der Stiftung Patientensicherheit ging man dieser Frage auf den Grund. die Tische der Verantwortlichen zu bringen. Dr. Priska Vonbach, Fachapothekerin und Abteilungsleiterin des Pharmazeutischen Dienstes im Universitäts-Kinderspital Zürich, «dachte bis zur Anfrage für das Referat, sie hätte nichts mit Design zu tun». Doch die Reflexion zu diesem Thema zeigte ihr einen neuen Blickwinkel. So stellt beispielsweise der schrittweise Umbau der Stationsapotheken zur Verbesserung der Medikationssicherheit nur einen Design-Aspekt ihres Alltags dar. Ziele dieser Anpassungen sind weniger Medikationsfehler durch helles Licht, weniger Lärm, Optimierung der Abläufe, Standardisierung sowie Optimierung der Temperatur. Denn sie weiss: "Jeden Kompromiss bezahlt man mit Prozesskosten mehrfach.»

\section{«Schlechtes Spitaldesign macht uns kränker.»}

Auch Dr. Anthony Staines, Verantwortlicher für die Patientensicherheitsprogramme der Fédération des hôpitaux vaudois, weiss, dass Licht- und Lärmverhältnisse zu ändern eine tiefhängende Frucht ist im Vergleich zur Veränderung von menschlichem Verhalten. Er berichtet über Studien zur Optimierung von Lichtund Lärmverhältnissen, die zu Verringerungen von unerwünschten Ereignissen führten. Es verwundert nicht, dass es zu Fehlern kommt, wenn sich Mitarbeitende zeitweise bei Lichtverhältnissen wie «4 Kerzen in der Nacht» oder beim Lärm eines «Lastwagenmotors» konzentrieren müssen. Dr. Dirk Hüske-Kraus, Clinical Director CTE - Medizintechnik von Philips, scherzt: «Bereits Gott wusste, dass die Trennung zwischen Licht und Finsternis gut war. So entstand das Paradies.» Ein Raum, der die Lichtverhältnisse der Natur imitiert, sei eine mögliche Lösung, um Patienten zu entlasten. Es gelte, die drei Dämonen der Intensivstation zu bekämpfen: Delir, Posttraumatische Belastungsstörung und die physische und psychische Belastung des Personals. Zwar hat die Überwachung und Alarmierung durch medizintechnische Geräte viele positive Effekte auf die Patientensicherheit. Gleichzeitig hat die Anzahl an Alarmen in den letzten Jahren aber enorm zugenommen. Eine Analyse hat gezeigt, dass diese bis zu 40\% reduziert werden könnten.

\section{Design darf nicht nur gut aussehen}

Dr. Rami Sommerstein, Oberarzt Infektiologie am Inselspital, macht darauf aufmerksam, dass beim Design immer auch Hygienefaktoren berücksichtigt werden müssen. Häufig liege das Problem darin, dass Geräte unter optimalen und damit realitätsfremden $\mathrm{Be}$ dingungen getestet würden. Geräte erweisen sich im schlimmsten Fall als Bakterienschleudern. Prof. Dr. 
Tom Guthknecht, Präsident der Lausanne Health and Hospitality Group, sieht die grösste Gefahr von gutem Design bei der Inkonsistenz. Das Thema Hygiene werde vernachlässigt - sowohl beim Bau von Spitälern als auch bei der Ausbildung von Ärzten und Spitalplanern.

\section{Offener Austausch und schrittweise Verbesserung}

In der Podiumsdiskussion, moderiert durch Dr. Yvonne Pfeiffer, reichen die Verbesserungsvorschläge von einer hierarchieübergreifenden Diskussions- und besseren Sicherheitskultur über finanzielle Incentivierung bis hin zum politischen Zwang. Da das Vertrauen der Bevölkerung in das Gesundheitswesen der Schweiz gross ist, fehlt der öffentliche Druck zur Verbesserung der Patientensicherheit. Teilweise fehlendes Problembewusstsein der Verantwortlichen und Kostenintransparenz verstärken das Problem. Hüske-Kraus glaubt zudem, dass holistische Ansätze überfordern. Eine schrittweise Verbesserung sei realistischer. Zimring schlägt vor, eine Austauschplattform einzurichten, bei dem alle Teilnehmer im Sinne eines gemeinsamen kontinuierlichen Verbesserungsprozesses gute und schlechte Erfahrungen teilen können. Die Studenten
Cornel Schiess und Monika Wyss setzen dabei auch auf die TeilnehmerInnen als Multiplikatoren. Prof. Dr. Dieter Conen wünscht sich «keine Dunkelkammer, sondern mehr Transparenz und weniger Konkurrenz».

\section{Bildnachweis}

๑) Stiftung Patientensicherheit Schweiz

Projektinformationen, Referate und Broschüre in Deutsch, Französisch, Italienisch und Englisch unter: http://www.patientensi cherheit.ch/de/themen/Patientensicherheit-Design.html

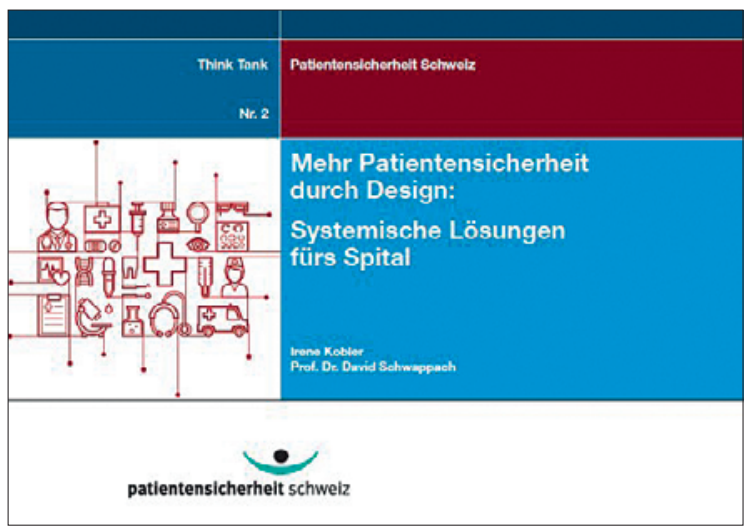

